\title{
Die Nicht-CPAP-Therapie des obstruktiven Schlafapnoe-Syndroms: ein Überblick
}

\author{
Non-CPAP Therapies in Obstructive Sleep Apnoea: An Overview
}

Autoren

Institute
S. Keymel' ${ }^{1}$ M. Kelm', W. J. Randerath ${ }^{2}$

${ }^{1}$ Heinrich-Heine Universität Düsseldorf, Medizinische Fakultät, Klinik für Kardiologie, Pneumologie und Angiologie, Universitätsklinikum Düsseldorf

${ }^{2}$ Institut für Pneumologie an der Universität Witten/Herdecke, Klinik für Pneumologie und Allergologie,

Zentrum für Schlaf- und Beatmungsmedizin, Krankenhaus Bethanien, Solingen

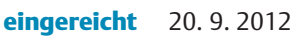
akzeptiert nach Revision 10. 10.2012

\section{Bibliografie}

Dol http://dx.doi.org/

10.1055/s-0032-1325903

Online-Publikation: 17.12 .2012

Pneumologie 2013; 67: 50-57

(c) Georg Thieme Verlag KG

Stuttgart · New York

ISSN 0934-8387

\section{Korrespondenzadresse}

Prof. Dr. med.

\section{Winfried J. Randerath}

Institut für Pneumologie an der Universität Witten/Herdecke, Klinik für Pneumologie und Allergologie, Zentrum für Schlaf- und Beatmungsmedizin, Krankenhaus Bethanien

Aufderhöher Straße 169-175 42699 Solingen

randerath@klinik-bethanien.de

\section{Zusammenfassung \\ $\nabla$}

Die optimale Therapie des obstruktiven Schlafapnoe-Syndroms erfordert ein individuell und interdisziplinär ausgerichtetes Konzept. Als Standardtherapie wird die nächtliche Überdruckatmung $(\mathrm{CPAP}=$ continuous positive airway pressure $)$ angesehen. Darüber hinaus existiert ein umfangreiches Repertoire an Nicht-CPAP-Therapien, die insbesondere bei Versagen oder Ablehnung der nächtlichen Überdruckatmung zum Einsatz kommen. Limitierend in der Bewertung der NichtCPAP-Therapie ist jedoch die vorwiegend unzureichende Evidenz der Nicht-CPAP-Therapien. Als konservative Maßnahme kann die Unterkieferprotrusionsschiene bei leichtem bis moderatem OSAS zum Einsatz kommen. Die bisher präklinisch eingesetzte Neurostimulation der Muskulatur der oberen Atemwege stellt einen attraktiven Ansatz zur Behandlung des OSAS dar, der sich jedoch in klinischen Studien noch beweisen muss. Isolierte chirurgische Maßnahmen der Nase, des Oropharynx oder des Hypopharynx können aufgrund der geringen Erfolgsrate im Allgemeinen nicht empfohlen werden. Ausnahmen stellen die Tonsillektomie bei Vergrößerung der Tonsillen und die aufwendige maxillomandibuläre Umstellungsosteotomie dar. Ein potenzieller Ansatz der chirurgischen Therapie des OSAS für die Zukunft stellt die Multi-Level-Chirurgie dar.

\section{Einleitung}

Das obstruktive Schlafapnoe-Syndrom (OSAS) stellt eine große Herausforderung in der Pneumologie dar. Dies beruht zum einen auf der hohen Prävalenz des OSAS von mindestens $2-4 \%$ [1], zum anderen auf den relevanten klinischen Folgeerscheinungen wie der reduzierten Lebensqualität, dem erhöhten Risiko für kardiovaskuläre und zerebrovaskuläre Erkrankungen sowie dem erhöhten Unfallrisiko. Die effektivste Therapie und

\section{Abstract \\ $\nabla$}

Optimal treatment of the obstructive sleep apnoea syndrome (OSAS) requires an individually designed and interdisciplinary approach. Continuous positive airway pressure (CPAP) is accepted as the first line therapy for patients with OSAS. However, non-CPAP therapies may be indicated as supportive therapeutical approach in CPAP failure or as an alternative approach in CPAP intolerance. Overall, the level of evidence for the majority of non-CPAP therapies is low. Mandibular advancement devices as a medical non-CPAP treatment have proven to reduce respiratory disturbances to a level which may be sufficient in mild to moderate sleep apnoea. Apnoea triggered neurostimulation of upper airway muscles is an innovative approach that has shown promising results in preclinical studies. Surgical treatment has previously been performed as single level surgery of the nasal, the oropharyngeal or hypopharyngeal level. However, only tonsillectomy in the presence of tonsillar hypertrophy and maxillomandibular advancement are recommended in carefully selected cases. Due to low success rates for single level surgery, multilevel surgery has been proposed as the surgical approach for the future.

somit die Standardtherapie des OSAS ist die nächtliche Überdruckatmung mittels CPAP (continuous positive airway pressure) [2-4]. Bei Ablehnung oder Versagen der CPAP-Therapie können Nicht-CPAP-Therapien zum Einsatz kommen. Als Allgemeinmaßnahme wird allen übergewichtigen Patienten eine Gewichtsreduktion empfohlen. Darüber hinaus können Nicht-CPAP-Therapien in medizinische und chirurgische Therapieansätze unterteilt werden. $\mathrm{Zu}$ den medizinischen Therapieansätzen zählen die Positionstherapie, 
Tab. 1 Übersicht von Nicht-CPAP-Therapien.

\begin{tabular}{|l}
\hline Allgemeine Maßnahmen \\
\hline - Schlafhygiene \\
\hline - Gewichtsreduktion \\
\hline Konservative Maßnahmen \\
\hline - Positionstherapie \\
\hline - Unterkieferprotrusionsschiene \\
\hline - Kieferorthopädie \\
\hline - Training der Muskulatur der oberen Atemwege \\
\hline - medikamentöse Therapie \\
\hline - interne oder externe Nasendilatatoren \\
\hline Operative Maßnahmen \\
\hline - Rhinochirurgie \\
\hline - Tonsillektomie/Tonsillotomie/Radiofrequenztherapie der Tonsillen \\
\hline - weicher Gaumen/Oropharynx \\
- Uvulopalatopharyngoplastie \\
- Laserassistierte Uvulopalatoplastie \\
- Radiofrequenztherapie des weichen Gaumens \\
- Uvulopalatale Flaps \\
- Weichteilgaumenimplantat \\
\hline - Zungenbasis/Hypopharynx \\
- Radiofrequenztherapie der Zungenbasis \\
- Hyoidsuspension \\
- Glossektomie entlang der Mittellinie \\
- Lingualplastik \\
- Entfernung der Zungenmandel \\
- Palatopharyngoglossoplastie \\
- Uvulopalatopharyngoglossoplastie \\
- Glossopexie \\
- Zungenbasissuspension \\
- Vorverlagerung des M. genioglossus \\
- Kieferchirurgie \\
- maxillomandibuläre Umstellungsosteotomie \\
- Distraktionsosteogenese \\
- Multi-Level-Chirurgie \\
\hline - Neurostimulation des N. hypoglossus \\
\hline
\end{tabular}

die Unterkieferprotrusionsschiene oder das Training der Muskulatur der oberen Atemwege. Die operativen Maßnahmen zeichnen sich vorwiegend durch das Therapieziel aus, die mechanische Obstruktion auf der Ebene der Nase, des Oropharynx oder des Hypopharynx zu beseitigen. Hieraus haben sich verschiedene operative Maßnahmen entwickelt, die als isolierte chirurgische Maßnahmen auf einer Ebene oder als Multi-Level-Chirurgie auf mehreren Ebenen der oberen Atemwege durchgeführt werden. Die Nicht-CPAP-Therapien des OSAS sind in der $\bullet$ Tab. 1 zusammengefasst.

\section{Pathophysiologie des obstruktiven Schlafapnoe- Syndroms}

Das Charakteristikum des OSAS ist die wiederholte partielle oder vollständige Obstruktion der oberen Atemwege trotz inspiratorischer Atembemühungen. Der hierdurch bedingte reduzierte oder unterbrochene Atemfluss resultiert in einer Sauerstoffentsättigung. Beendet wird das Ereignis durch eine Weckreaktion mit der Folge einer Schlaffragmentation. Die Obstruktion der oberen Atemwege ist ein multifaktorielles Geschehen [5]. Eine vermehrte Kollapsneigung der oberen Atemwege wird als eine Ursache für das OSAS angesehen [6]. Die Kollapsneigung wird u.a. durch die Anatomie der oberen Atemwege beeinflusst. Typischerweise ist der Querschnitt der oberen Atemwege bei Patienten mit OSAS im Retropalateal- und/oder im Retroglossalbereich eingeengt. Bildgebende Verfahren konnten darstellen, dass bei OSAS-Patienten vorzugsweise die laterale Achse der oberen Atemwege durch eine Verdickung der lateralen Pharyngealwände vermindert ist $[7,8]$. Darüber hinaus tragen eine Vergrößerung der Tonsillen, der Uvula, des Gaumens oder der Zunge zu der Obstruktion bei $[7,8]$. Auch knöcherne Strukturen wie Unterkiefer oder Zungenbein beeinflussen die Weite des Atemwegsquerschnitts [9]. Zusätzlich tragen funktionelle Veränderungen zu der Obstruktion der oberen Atemwege bei. Die während des Schlafs auftretende Muskelrelaxation kann zu einer relevanten Einengung der oberen Atemwege führen. Entscheidend für den Kollaps der Atemwege ist hierbei das Gleichgewicht zwischen dem negativen intrapharyngealen Druck und der gegensätzlich wirkenden Kraft der dilatierenden Muskulatur [10]. Methodisch ist die Untersuchung der Aktivität der Muskulatur der oberen Atemwege jedoch schwierig, sodass hierzu nur wenige humane Studien vorliegen. Es konnte gezeigt werden, dass der M. genioglossus als relevanter dilatierender Muskel der oberen Atemwege eine kompensatorisch erhöhte Aktivität bei wachen OSAS-Patienten im Vergleich zu Kontrollpatienten zeigte [11]. Es wird vermutet, dass dieser Mechanismus jedoch während des Schlafs versagt [11]. Hierzu tragen möglicherweise neurogene Läsionen der afferenten und efferenten Nerven bei [12]. Als mögliche Ursache wird von den Autoren die Kombination aus einem Vibrationsschaden und Hypoxämie diskutiert [12].

\section{Klinische Folgen des OSAS}

Tagesschläfrigkeit bis hin zum unfreiwilligen Einschlafen ist das führende Symptom des OSAS. Als weiterer Hauptbefund werden fremdanamnestisch Atemstillstände berichtet. Darüber hinaus können nächtliches Aufschrecken mit kurzzeitiger Atemnot, Schnarchen, insomnische Beschwerden mit häufigem nächtlichen Erwachen, nächtliche Palpitationen, Nykturie, Nachtschweiß, Enuresis, morgendliche Schlaftrunkenheit und nächtliche bzw. morgendliche Kopfschmerzen auftreten [4].

Schlafbezogene Atmungsstörungen sind mit einem erhöhten Unfallrisiko assoziiert [13]. Patienten mit einem Apnoe-HypopnoeIndex $(\mathrm{AHI})>15 /$ Stunde hatten ein 7-fach erhöhtes Risiko mehrfacher Unfälle in 5 Jahren im Vergleich zu Probanden ohne schlafbezogene Atmungsstörung [13]. Außerdem wurde OSAS als ein unabhängiger Risikofaktor für kardiovaskuläre und zerebrovaskuläre Erkrankungen identifiziert [14,15]. Das OSAS stellt einen relevanten Risikofaktor für die Entwicklung einer arteriellen Hypertonie dar [16]. Entsprechend sollte ein OSAS im Rahmen der Diagnostik einer arteriellen Hypertonie zum Ausschluss einer sekundären Form berücksichtigt werden, wie dies auch in den Leitlinien für das Management der arteriellen Hypertonie empfohlen wird [17]. OSAS erhöht außerdem das Risiko für eine koronare Herzkrankheit (KHK) $[14,15]$. In der Sleep Heart Study wurde der Zusammenhang zwischen dem AHI und selbst berichteten kardiovaskulären Ereignissen untersucht. Hier zeigte sich bei $16 \%$ der Teilnehmer eine kardiovaskuläre Erkrankung. Nach Unterteilung des AHI in Quartile zeigte sich eine zunehmende Häufigkeit der KHK mit 9\% in der 1. Quartile (AHI 0-1,3/h) und $19 \%$ in der 4. Quartile (AHI>11/h) [14]. Hierbei ist zu beachten, dass ein ungünstiger Verlauf der KHK wie „Major adverse cardiac events“ [18], Restenosen [18,19] und eine erhöhte Mortalität [20] mit einem erhöhten AHI assoziiert sind. Darüber hinaus zeigt sich bei OSAS-Patienten ein erhöhtes Risiko für Herzrhythmusstörungen wie Vorhofflimmern [21-23], Blockbilder [24] oder komplexe ventrikuläre Extrasystolie [23]. In diesem Zusammenhang gelangt die Komorbidität von OSAS und Vorhofflimmern zunehmend in das Interesse der Pneumologie und Kardiologie. Bei Vorliegen von OSAS ist die Prävalenz von Vorhofflim- 
mern um den Faktor 2 - 3 erhöht [23, 25]. Zudem scheint das Vorliegen eines OSAS den Erfolg therapeutischer Maßnahmen bei Vorhofflimmern zu beeinflussen. Das Vorliegen eines OSAS erhöht das Rezidivrisiko von Vorhofflimmern/-flattern nach elektrischer Kardioversion [26] oder Pulmonalvenenisolation [22].

\section{Die Nicht-CPAP-Therapie des obstruktiven Schlafapnoe-Syndroms \\ $\nabla$}

Die effektivste Therapie für alle Schweregrade des OSAS ist die nächtliche Überdruckatmung (CPAP = continuous positive airway pressure) $[4,27,28]$. Entsprechend sollte die nächtliche Überdruckatmung Patienten mit OSAS als primäre Therapie angeboten werden. Zusätzlich existieren verschiedene Nicht-CPAP-Therapien. Diese können in konservative und chirurgische Therapieansätze eingeteilt werden ( Tab. 1). Im Folgenden sollen die relevanten Therapieansätze beschrieben und in Anlehnung an die S3-Leitlinie „Nicht-erholsamer Schlaf/Schlafstörungen“ [4], die S2-Leitlinie "Therapie der Schlafapnoe des Erwachsenen“ [29] und an den Task Force Report der European Respiratory Society bewertet werden [30].

\section{Konservative Maßnahmen \\ $\nabla$}

\section{Gewichtsreduktion}

Übergewicht bzw. Adipositas gelten als relevante Risikofaktoren für das OSAS [31,32]. Peppard und Kollegen haben in einer longitudinalen Beobachtungsstudie gezeigt, dass eine Gewichtszunahme von $10 \%$ das Risiko für ein moderates bis schweres OSAS um den Faktor 6 erhöht [31]. Es wird vermutet, dass Übergewicht zu strukturellen und funktionellen Veränderungen führt, die eine vermehrte Kollapsneigung der oberen Atemwege zur Folge haben [33]. Hierbei scheinen zum einen Fetteinlagerungen in den oberen Atemwegen, zum anderen zentrale Fetteinlagerungen über eine Reduktion der funktionellen Residualkapazität von Relevanz zu sein. Entsprechend wird allen übergewichtigen Patienten mit OSAS als allgemeine Empfehlung eine Gewichtsreduktion als Begleittherapie empfohlen [4,30,34]. Es konnte gezeigt werden, dass bei übergewichtigen Patienten mit OSAS mit einer durchschnittlichen Gewichtsreduktion von $10 \mathrm{~kg}$ durch Änderung des Lebensstils und diätetische Maßnahmen eine Abnahme des AHI um 4-5 Ereignisse/Stunde erreicht werden kann $[35,36]$. Bariatrische Chirurgie sollte einzelnen Fällen mit morbider Adipositas vorbehalten sein, insbesondere vor dem Hintergrund, dass die CPAP-Therapie effektiver in der Behandlung des OSAS ist als eine Gewichtsreduktion [37]. Für eine operative Maßnahme spricht über die Behandlung des OSAS hinaus der langfristige Erfolg weiterer klinischer Folgen der Adipositas, die entsprechend in der Entscheidung berücksichtigt werden sollten.

\section{Positionstherapie}

Die Schlafposition kann einen Einflussfaktor auf die Schwere des OSAS darstellen [38]. Bei einem Teil der Patienten mit OSAS konnte eine Zunahme des AHI in Rückenlage im Vergleich zur Seitenlage im Schlaf beobachtet werden [38]. Die Prävalenz des Rückenlage-assoziierten OSAS beträgt ca. 55\% [39]. Bei Patienten mit Rückenlage-assoziiertem OSAS wurde eine Positionstherapie mittels verschiedener Hilfsmittel wie dem Tennisball-Gurt [40] oder der Rückenlage-Verhinderungsweste [41,42] versucht. Es konnte gezeigt werden, dass durch die Vermeidung der Rückenlage der AHI [40-42] und die Tagesschläfrigkeit [41] reduziert werden kann. Es ist jedoch ausdrücklich zu betonen, dass die Positionstherapie häufig das OSAS nicht vollständig behandelt [40 - 42]. Entsprechend kann die Positionstherapie als alleinige Therapie des OSAS nicht empfohlen werden. Sollte die Positionstherapie in Einzelfällen eingesetzt werden, muss eine polysomnografische Kontrolle zur Dokumentation des Therapieerfolgs erfolgen [30].

\section{Unterkieferprotrusionsschienen}

Die Unterkieferprotrusionsschiene (UPS) ist ein intraorales Device, das zu einer Vorschubposition des Unterkiefers während des Schlafs führt $[43,44]$. Hierdurch wird der Atemwegsquerschnitt der oberen Atemwege auf der retroglossalen und retropalatealen Ebene mechanisch vergrößert $[45,46]$ und die Kollapsneigung des Pharynx vermindert [47]. Es existiert eine Vielzahl verschiedener UPS auf dem Markt. Generell sollten adjustierbare, individuell durch schlafmedizinisch geschulte Zahnärzte angefertigte UPS eingesetzt werden [48]. Zweischienensysteme sollten gegenüber Monoblock-Systemen bevorzugt werden. Die individuelle Einstellung des Unterkiefervorschubs, der anhand eines Titrierprozesses in einem Schlaflabor festgelegt wird, ist entscheidend für den Therapieerfolg $[49,50]$. Typischerweise beträgt der Vorschub des Unterkiefers 6-10 mm oder 50-75\% des maximalen Unterkiefervorschubs [51]. Die vertikale Öffnung des UPS scheint von untergeordneter Bedeutung zu sein [52] ( $\bullet$ Abb.1a-d).

Die Effektivität von UPS konnte in randomisierten Studien belegt werden. Im Vergleich zu einer Plazebo-Behandlung kann durch den Einsatz von UPS eine Reduktion des AHI erreicht werden [44, 51,53,54]. Die durchschnittliche Erfolgsrate in der Behandlung des OSAS durch UPS beträgt $42 \%$, wenn man Erfolg als einen AHI $<5 /$ Stunde definiert, und $52 \%$, wenn man Erfolg als einen AHI $<10$ definiert [51]. Die Erfolgsraten sind höher bei Patienten mit mildem OSAS [54,55]. Weitere Prädiktoren für einen Therapieerfolg von UPS sind junges Alter [56], normales Gewicht [56], weibliches Geschlecht [54], Rückenlage-assoziertes OSAS [54] sowie bestimmte cephalometrische Maße [56]. Im Vergleich zur CPAP-Therapie sind UPS insgesamt in der Reduktion des AHI jedoch unterlegen [43,57-61]. Nur bei leichtem OSAS können vergleichbare Ergebnisse erreicht werden [55] und stellen somit hier eine echte Alternative zur CPAP-Therapie dar.

Typische Nebenwirkungen der UPS sind Zahn- und/oder Kieferschmerzen, zeitweise morgendliche Schlussunfähigkeit des Mundes und exzessive Hypersalivation [43,57-59]. Die Nebenwirkungen der UPS erklären z.T. die nicht optimale Therapieadhärenz. Es wurde beobachtet, dass 76\% der Patienten die Therapie mit dem UPS über 1 Jahr [54] und 65\% über 4 Jahre [62] fortgesetzt haben. Im Vergleich zur CPAP-Therapie scheinen die Patienten die UPS zu bevorzugen [43,57,58,61].

Zusammenfassend konnte gezeigt werden, dass durch den Einsatz von UPS die Häufigkeit obstruktiver Ereignisse reduziert werden konnte. Es wird hierbei empfohlen, individuell angefertigte adjustierbare Devices einzusetzen, deren optimale Einstellung nach einem Titrationsprozess polysomnografisch überprüft werden sollte. UPS stellen eine Therapiealternative zu CPAP bei leichtem bis mittelschwerem OSAS dar. Bei schwerem OSAS können UPS bei Ablehnung oder Versagen der CPAP-Therapie erwogen werden $[4,30,63]$. 

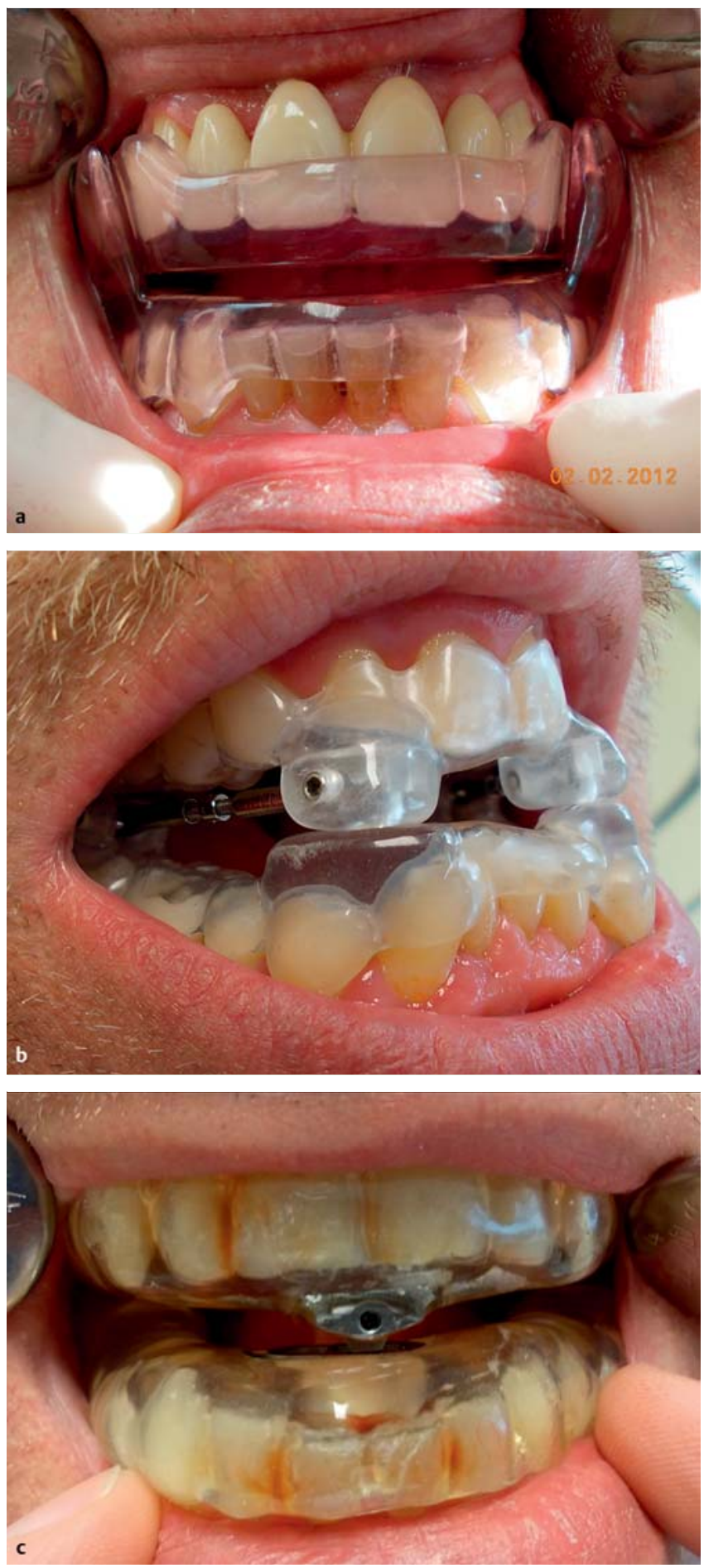

\section{Kieferorthopädie}

Das OSAS bei Kindern kann bei Vorliegen von bestimmten Fehlstellungen wie z. B. dem Kreuzbiss mittels früher kieferorthopädischer Eingriffe behandelt werden [64, 65]. Zu den kieferorthopädischen Eingriffen zählt die forcierte Gaumennahterweiterung, die durch eine laterale Erweiterung des Oberkiefers eine Vergrößerung des Nasen- und Oropharyngealraums bewirken soll. Diese Maßnahme ist bei Kindern möglich, da die Gaumennaht, an der die beiden Oberkieferhälften verbunden sind, noch nicht verknöchert ist. Für die forcierte Gaumennahterweiterung wird über einen Zeitraum von 12 Monaten ein intraorales Device an den Zähnen des Oberkiefers befestigt. In der ersten Phase wird über

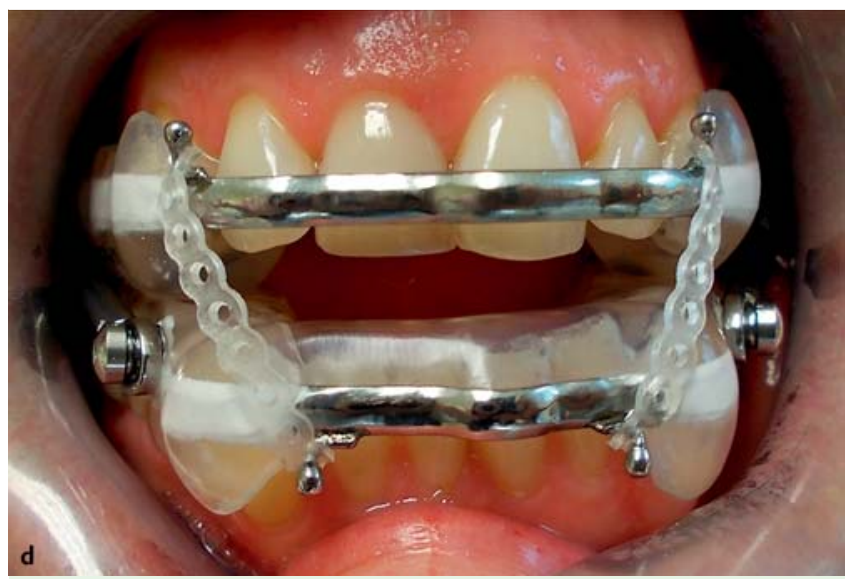

Abb. 1 Die Abbildungen $\mathbf{1}$ a-d zeigen verschiedene Unterkieferprotrusionsschienen-Systeme (UPS-Systeme): 1B - Hamburger UPS (H-UPS soft) nach Schlieper; 1B-ISTplus nach Hinz aus LAMItec; 1C-TAP soft; 1D-SomnoDent soft (mit up-down Gummizügen); 1E-SomnoDent soft. Alle abgebildeten Hilfsmittel wurden individuell angefertigt. Es handelt sich um Zwei-Schienensysteme (Ober- und Unterkieferteil). Die Systeme sind individuell, entsprechend dem Therapieeffekt, adjustierbar.

ein Schraubensystem täglich eine Druckerhöhung und somit eine Erweiterung der Gaumennaht bewirkt. In der zweiten Phase wird das Device in konstanter Position belassen, um eine Distraktionsosteogenese zu ermöglichen. Es konnte gezeigt werden, dass durch die forcierte Gaumennahterweiterung eine effektive Reduktion des AHI erreicht werden kann $[64,65]$. Der Erfolg scheint längerfristig anzuhalten [66]. Bei jungen Erwachsenen liegen Fallberichte vor, dass eine forcierte Gaumennahterweiterung erfolgreich in der Behandlung des OSAS eingesetzt wurde [67].

\section{Training der Muskulatur der oberen Atemwege}

Als eine Ursache in der Entstehung des OSAS wurde eine verminderte neuromuskuläre Aktivität der dilatierenden Muskeln der oberen Atemwege gefunden [10]. Basierend auf dieser Tatsache wurden verschiedene Ansätze zur Überwindung der muskulären Dysfunktion untersucht. Ein Ansatz ist ein Training der Atemwegsmuskulatur durch spezielle oropharyngeale Übungen [68] oder Didgeridoo spielen [69], wodurch eine gewisse Reduktion des AHI erreicht werden kann. Ein Training der oberen Atemwege kann alternativ durch eine regelmäßige elektrische Stimulation der Zungenmuskulatur erfolgen [70]. Hierdurch kann insbesondere eine Reduktion des Schnarchens erreicht werden [70]. Als weiterer Ansatz ist die gezielte Stimulation des M. genioglossus als dem stärksten dilatierenden Muskel der oberen Atemwege oder des N. hypoglossus während des Schlafes entwickelt worden $[71,72]$. Es konnte in präklinischen Studien gezeigt werden, dass die intraneurale Stimulation des Nervus hypoglossus oder die transkutane Stimulation des M. genioglossus zu einer Verbesserung des AHI führt [71,72]. Hierbei ist zu berücksichtigen, dass die transkutane Stimulation mit Missempfindungen durch die Stimulation verbunden ist. Die positiven Ergebnisse der Neurostimulation des Nervus hypoglossus führten zur Entwicklung implantierbarer Stimulationssysteme, die durch eine atemsynchrone elektrische Stimulation des Nervus hypoglossus eine Erweiterung der oberen Atemwege während des Schlafes bewirken sollen ( $\bullet$ Abb. 2) [73]. Es konnte gezeigt werden, dass Patienten mit einem $\mathrm{BMI}<32 \mathrm{~kg} / \mathrm{m}^{2}$, einem $\mathrm{AHI}<50 /$ Stunde und einem nicht vollständigen palatealen Kollaps von der Therapie profitieren können [73]. Bei 8 Patienten mit den genannten Responder- 


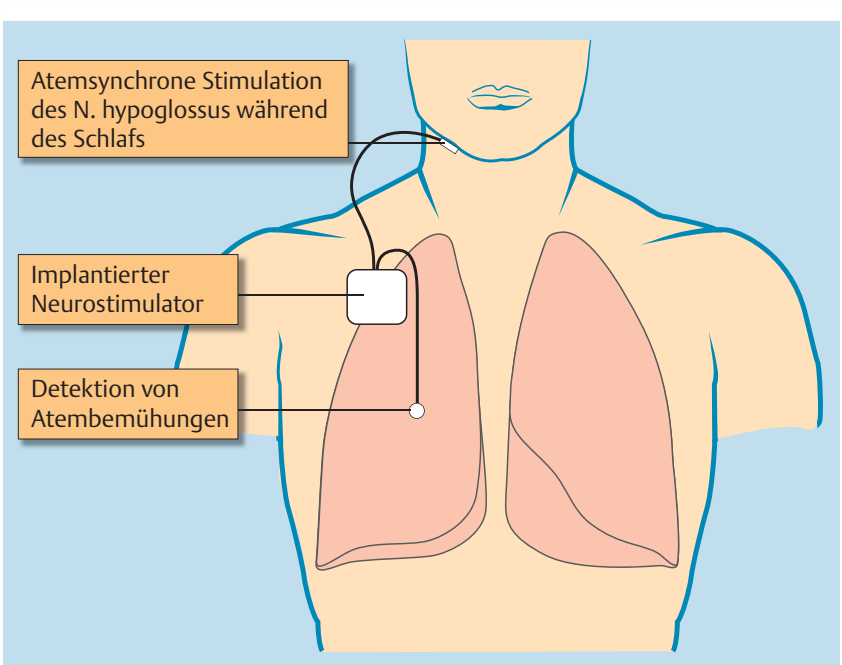

Abb.2 Implantierbares Schrittmachersystem zur Stimulation des Musculus genioglossus. Die Abbildung zeigt schematisch den Aufbau eines Systems zur Stimulation des Nervus hypoglossus mittels eines implantierbaren Schrittmachersystems (modifiziert nach [73]). Das System besteht aus einem Sensor, der die Atemanstrengung erfasst und somit die Inspiration erkennen lässt. Eine Elektrode wird an den Teil des Nervus hypoglossus angelegt, der die dilatatorischen Fasern des Musculus genioglossus versorgt. Das Schrittmachersystem appliziert Stimulationsimpulse bei jeder detektierten Inspirationsbemühung. Der Patient kann das System einschalten, wenn er sich zum Schlafen hinlegt und nach dem Erwachen wieder deaktivieren.

Kriterien konnte eine Reduktion des AHI von 40/Stunde auf 10/ Stunde erreicht werden [73]. Bis dato kann aufgrund der geringen Datenlage der jungen Methode die neuromuskuläre Stimulation noch nicht endgültig empfohlen werden. Die neuromuskuläre Stimulation stellt allerdings eine vielversprechende Therapieoption für die Zukunft dar [30].

\section{Operative Maßnahmen}

Nase

Eine isolierte rhinochirurgische Maßnahme wie die Nasenseptumplastik, Nasenmuschelplastik, Septorhinoplastik oder Nasennebenhöhlen-Operationen wird zur Behandlung des OSAS im Allgemeinen nicht empfohlen, da dies nicht zu einer effektiven Behandlung des OSAS führt $[29,30]$. Bei CPAP-Versagen aufgrund einer nasalen Obstruktion oder als ein Element der Multi-LevelChirurgie kann allerdings eine rhinochirurgische Maßnahme in Einzelfällen sinnvoll sein.

\section{Tonsillen}

Eine substanzielle Vergrößerung der Tonsillen kann zu einer Obstruktion der oberen Atemwege beitragen. Die Größe der Tonsillen ist mit der Schwere des OSAS assoziiert [74]. Bei Patienten mit CPAP-Versagen und einer pharyngealen Obstruktion durch eine Vergrößerung der Tonsillen kann durch eine Tonsillektomie eine Verbesserung des OSAS erreicht werden $[75,76]$ und der erforderliche CPAP-Druck reduziert werden [76]. In Anbetracht des niedrigen Operationsrisikos einer Tonsillektomie kann die Operation in diesen Fällen indiziert sein $[29,30]$. Hierbei sollte eine klassische Tonsillektomie durchgeführt werden. Aufgrund der unzureichenden Datenlage sowie des Rezidivrisikos sind die Tonsillotomie und die interstitielle Radiofrequenztherapie nicht als gleichwertige Therapie anzusehen $[29,30]$. Bei kindlichem OSAS wird eine Adenotonsillektomie empfohlen [30].

\section{Weicher Gaumen}

Eine Obstruktion der oberen Atemwege auf der retropalatealen Ebene ist eine häufige Ursache des OSAS. Die klassische Operationsmethode zur Beseitigung einer retropalatealen Obstruktion ist die Uvulopalatopharyngoplastie (UPPP). Die UPPP zählt zu den resektiven chirurgischen Maßnahmen. Das Prinzip besteht in der Entfernung überschüssiger Schleimhaut im Bereich des Weichgaumens und der Uvula, ohne die Muskulatur, insbesondere die Muskulatur des vorderen Gaumenbogens, zu schädigen. Weiterhin soll die Funktion des Weichgaumens erhalten bleiben $[29,30,77]$. Eine muskelschonende Operationstechnik ist von entscheidender Bedeutung zur Vermeidung postoperativer funktioneller Störungen, insbesondere des Schluckens oder der Phonation [78]. Die UPPP wird häufig mit einer Tonsillektomie kombiniert $[78,79]$. In einer Metaanalyse von Elshaug et al. zeigte sich, dass die Erfolgsrate der UPPP 52\% beträgt, wenn Erfolg als eine Reduktion des AHI um 50\% und/oder auf einen AHI $<20 /$ Stunde definiert ist [80]. Definiert man allerdings Erfolg als $\mathrm{AHI}<5 /$ Stunde, beträgt die Erfolgsrate nur 16\% [80]. Vergleichsstudien zur CPAP-Therapie liegen nicht vor. Im Vergleich zur UPS ist die UPPP unterlegen [81]. Neben der geringen Erfolgsquote sowie dem fehlenden Vergleich mit der Standardtherapie ist bei der Bewertung der UPPP außerdem das Risiko peri- und postoperativer Komplikationen zu beachten. Die Rate an ernsten nichtfatalen Komplikationen beträgt 1,5\%, die Mortalität 0,2\% [82]. Die UPPP kann insgesamt nur in Einzelfällen nach exakter diagnostischer Aufarbeitung mit Nachweis einer relevanten retropalatealen Obstruktion empfohlen werden $[29,30]$.

Modifizierte Verfahren wie die laserassistierte Uvulopalatoplastie, uvulopalateale Flaps oder Radiofrequenztherapie des weichen Gaumens können aufgrund der geringen Evidenz bzw. der geringen Erfolgsrate als isolierte operative Maßnahme nicht empfohlen werden $[29,30,80]$. Weichteilgaumenimplantate [83] können in Einzelfällen bei leichtem bis moderatem OSAS erwogen werden, wenn konservative Maßnahmen abgelehnt werden $[29,30]$.

\section{Zungengrund und Hypopharynx}

Verschiedene chirurgische Therapieansätze zielen auf eine Beseitigung einer retrolingualen Obstruktion ab. Zur Radiofrequenztherapie des Zungengrundes liegt eine randomisierte, kontrollierte Studie vor, in der die Radiofrequenztherapie des Zungengrundes und des weichen Gaumens mit CPAP-Therapie verglichen wurde [84]. Es konnte gezeigt werden, dass die Radiofrequenztherapie zu einer Verbesserung der Lebensqualität führte, die mit der CPAP-Therapie vergleichbar war. Bezüglich des AHI war die Radiofrequenztherapie jedoch der CPAP-Therapie unterlegen [84]. Weitere chirurgische Maßnahmen des Zungengrundes wie die Teilresektion des Zungengrundes, die laserassistierte Glossektomie entlang der Mittellinie, die Glossopexie oder die Hyoidsuspension können als isolierte Operation ebenfalls aufgrund der geringen Datenlage und/oder niedrigen Erfolgsrate nicht empfohlen werden, haben aber eine mögliche Bedeutung als Element in der Multi-Level-Chirurgie [29,30,85].

\section{Kieferchirurgische Maßnahmen}

\section{Maxillomandibuläre Umstellungsosteotomie}

Die maxillomandibuläre Umstellungsosteotomie (MMO) ist ein aufwendiges operatives Verfahren, das durch eine Vorverlagerung des Unterkiefers zu einer Vergrößerung des retrolingualen und retropalatealen Atemwegs führen soll. Bei dem klassischen Operationsverfahren erfolgte eine beidseitige sagittale Spaltung 
des Unterkieferknochens in Kombination mit einer Le-Fort-I-Osteotomie des Oberkiefers $[86,87]$. Es hat sich gezeigt, dass die MMO ein effektives Verfahren in der Behandlung des Schlafapnoesyndroms ist $[86,87]$. Der Therapieerfolg scheint vergleichbar mit der CPAP-Therapie zu sein [88]. Im Gegensatz zu anderen operativen Maßnahmen scheint bei der MMO der Erfolg längerfristig anhaltend zu sein [89]. Trotz der positiven Ergebnisse der MMO sollten jedoch die peri- und postoperativen Komplikationen der aufwendigen Operation im Sinne einer Risiko-NutzenBewertung beachtet werden. Die MMO kann entsprechend in Einzelfällen bei Ablehnung der nächtlichen Beatmungstherapie und maxillomandibulären Anomalien nach sorgfältiger cephalometrischer Untersuchung empfohlen werden [29,30].

\section{Distraktionsosteogenese}

Die Distraktionsosteogenese (DOG) wird üblicherweise bereits im Kindesalter bei angeborenen Fehlbildungen des Mittelgesichts oder Mikrognathie durchgeführt [90,91]. Hierbei erfolgt zunächst eine Osteotomie des Ober- oder Unterkieferknochens und im Anschluss eine langsame Distraktion der Knochensegmente über mehrere Wochen. Als primäres Therapieziel wird hierbei die Vermeidung einer Tracheotomie angesehen. Es liegen Fallberichte vor, die darauf hinweisen, dass eine DOG auch bei Erwachsenen mit Schlafapnoe-Syndrom erfolgreich sein kann [92, 93], sodass die DOG bei Patienten mit Mikrognathie oder Hypoplasie des Mittelgesichts erwogen werden kann.

Zusammenfassend gibt es eine Vielzahl isolierter chirurgischer Verfahren. Die Bewertung der einzelnen Verfahren ist insofern erschwert, dass belastbare Studiendaten bezüglich der Erfolgsrate sowie des Vergleichs mit der Standardtherapie des OSAS, der nächtlichen Überdruckatmung mittels CPAP, weitestgehend fehlen. Als isolierte chirurgische Maßnahme können letztendlich nur die Tonsillektomie bei Vergrößerung der Tonsillen und die MMO bei maxillomandibulären Anomalien über den Einzelfall hinaus empfohlen werden.

\section{Multi-Level-Chirurgie}

Nachdem sich die isolierten chirurgischen Eingriffe als nicht zufriedenstellend in der Behandlung des OSAS gezeigt haben, ist das Konzept der Multi-Level-Chirurgie (MLS) in den Vordergrund gerückt. Als Initiatoren können Fujita et al. [94] sowie Riley et al. [95] mit Nachweis sowie der Einteilung einer Obstruktion der oberen Atemwege auf mehreren Ebenen bei der Mehrzahl der Patienten mit OSAS angesehen werden. Eine exakte Definition der Multi-Level-Chirurgie existiert nicht. Am häufigsten wird mindestens ein Eingriff am Zungengrund/Hypopharynx mit mindestens einem Eingriff an Weichgaumen/Tonsillen kombiniert $[29,85]$. Die Erfolgsrate der Multi-Level-Chirurgie scheint mit $66 \%$ höher zu sein als bei der Single-Level-Chirurgie [85]. Auf der anderen Seite der Medaille ist jedoch eine hohe Komplikationsrate von ca. 15\% zu beachten [85]. Insgesamt kann zum aktuellen Zeitpunkt die Multi-Level-Chirurgie nicht als Ersatz für eine nächtliche Überdruckatmung zur Behandlung des OSAS empfohlen werden. Die Multi-Level-Chirurgie kann allerdings eine Alternative bei Patienten mit CPAP-Versagen oder -Ablehnung darstellen.

\section{Ausblick}

$\nabla$

Aufgrund der Komplexität der OSAS erfordert eine optimale Therapie eine auf den Patienten individuell zugeschnittene und interdisziplinäre Strategie. Bei Ablehnung oder Versagen der CPAP-Therapie steht ein umfangreiches Repertoire an NichtCPAP-Therapie zur Verfügung. Aufgrund der insgesamt geringen Evidenz der Nicht-CPAP-Therapien sind klinische Studien zur Wirksamkeit im Vergleich zur CPAP-Therapie wünschenswert. Die Neurostimulation der N. hypoglossus hat sich in präklinischen Studien als wirkungsvoll erwiesen, sodass dieses Konzept einen vielversprechenden Ansatz für die Zukunft darstellt. Aus chirurgischer Sicht scheint die Multi-Level-Chirurgie den isolierten operativen Maßnahmen überlegen zu sein.

\section{Danksagung \\ $\nabla$}

Die Autoren danken Dr. med. dent. Alexander Meyer für die Zurverfügungstellung der Abbildungen verschiedener UPS-Systeme.

\section{Interessenkonflikt \\ $\nabla$}

S. Keymel und M. Kelm geben an, dass kein Interessenkonflikt besteht.

W. Randerath hat in den vergangenen 5 Jahren Vortragshonorare, Beraterhonorare und Reisekostenerstattungen der Firmen Weinmann, Resmed, Respironics erhalten.

\section{Literatur}

1 Young T, Palta M, Dempsey J et al. The occurrence of sleep-disordered breathing among middle-aged adults. N Engl J Med 1993; 328: $1230-1235$

2 Basner RC. Continuous positive airway pressure for obstructive sleep apnea. N Engl J Med 2007; 356: 1751-1758

3 Gay P, Weaver T, Loube D et al. Evaluation of positive airway pressure treatment for sleep related breathing disorders in adults. Sleep 2006; 29: $381-401$

4 Deutsche Gesellschaft für Schlafforschung und Schlafmedizin (DGSM). S3-Leitlinie Nicht erholsamer Schlaf/Schlafstörungen. Somnologie 2009; 13 (Suppl. 01): 4-160

5 Eckert DJ, Malhotra A, Jordan AS. Mechanisms of apnea. Prog Cardiovasc Dis 2009; 51: 313-323

6 Malhotra A, Pillar G, Fogel R et al. Upper-airway collapsibility: measurements and sleep effects. Chest 2001; 120: 156-161

7 Schellenberg JB, Maislin G, Schwab RJ. Physical findings and the risk for obstructive sleep apnea. The importance of oropharyngeal structures. Am J Respir Crit Care Med 2000; 162: 740 - 748

8 Schwab RJ, Pasirstein M, Pierson R et al. Identification of upper airway anatomic risk factors for obstructive sleep apnea with volumetric magnetic resonance imaging. Am J Respir Crit Care Med 2003; 168: 522 530

$9 \mathrm{Yu}$ X, Fujimoto K, Urushibata $\mathrm{K}$ et al. Cephalometric analysis in obese and nonobese patients with obstructive sleep apnea syndrome. Chest 2003; 124: $212-218$

10 Remmers JE, deGroot WJ, Sauerland EK et al. Pathogenesis of upper airway occlusion during sleep. J Appl Physiol 1978; 44: 931 -938

11 Mezzanotte WS, Tangel DJ, White DP. Waking genioglossal electromyogram in sleep apnea patients versus normal controls (a neuromuscular compensatory mechanism). J Clin Invest 1992; 89: 1571 - 1579

12 Saboisky JP, Stashuk DW, Hamilton-Wright A et al. Neurogenic changes in the upper airway of patients with obstructive sleep apnea. Am J Respir Crit Care Med 2012; 185: 322 - 329

13 Young T, Blustein J, Finn L et al. Sleep-disordered breathing and motor vehicle accidents in a population-based sample of employed adults. Sleep 1997; 20: 608-613 
14 Shahar E, Whitney CW, Redline S et al. Sleep-disordered breathing and cardiovascular disease: cross-sectional results of the Sleep Heart Health Study. Am J Respir Crit Care Med 2001; 163: 19-25

15 Peker Y, Carlson J, Hedner J. Increased incidence of coronary artery disease in sleep apnoea: a long-term follow-up. Eur Respir J 2006; 28 : 596-602

16 Peppard PE, Young T, Palta $M$ et al. Prospective study of the association between sleep-disordered breathing and hypertension. N Engl J Med 2000; 342: 1378 - 1384

17 Mancia G, De Backer G, Dominiczak A et al. 2007 Guidelines of the management of arterial hypertension. The task force of the management of arterial hypertension of the european society of hypertension (ESH) and the european society of cardiology (ESC). Eur Heart J 2007; 28: $1462-1536$

18 Yumino D, Tsurumi Y, Takagi A et al. Impact of obstructive sleep apnea on clinical and angiographic outcomes following percutaneous coronary intervention in patients with acute coronary syndrome. Am J Cardiol 2007; 99: 26-30

19 Steiner S, Schueller PO, Hennersdorf MG et al. Impact of obstructive sleep apnea on the occurrence of restenosis after elective percutaneous coronary intervention in ischemic heart disease. Respir Res 2008; 9: 50

20 Peker Y, Hedner J, Kraiczi H et al. Respiratory disturbance index: an independent predictor of mortality in coronary artery disease. Am J Respir Crit Care Med 2000; 162: 81-86

21 Gami AS, Pressman G, Caples SM et al. Association of atrial fibrillation and obstructive sleep apnea. Circulation 2004; 110: 364-367

$22 \mathrm{Ng} \mathrm{CY}$, Liu T, Shehata M et al. Meta-analysis of obstructive sleep apnea as predictor of atrial fibrillation recurrence after catheter ablation. Am J Cardiol 2011; 108: 47-51

23 Mehra R, Stone KL, Varosy PD et al. Nocturnal Arrhythmias across a spectrum of obstructive and central sleep-disordered breathing in older men: outcomes of sleep disorders in older men (MrOS sleep) study. Arch Intern Med 2009; 169: 1147-1155

24 Koehler U, Fus E, Grimm W et al. Heart block in patients with obstructive sleep apnoea: pathogenetic factors and effects of treatment. Eur Respir J 1998; 11: 434-439

25 Stevenson IH, Teichtahl H, Cunnington D et al. Prevalence of sleep disordered breathing in paroxysmal and persistent atrial fibrillation patients with normal left ventricular function. Eur Heart J 2008; 29 : $1662-1669$

26 Kanagala R, Murali NS, Friedman PA et al. Obstructive sleep apnea and the recurrence of atrial fibrillation. Circulation 2003; 107: 2589-2594

27 Kushida CA, Littner MR, Hirshkowitz M et al. Practice parameters for the use of continuous and bilevel positive airway pressure devices to treat adult patients with sleep-related breathing disorders. Sleep 2006; 29 : $375-380$

28 Giles TL, Lasserson TJ, Smith BH et al. Continuous positive airways pressure for obstructive sleep apnoea in adults. Cochrane Database Syst Rev 2006: CD001106

29 Verse T, Bodlaj R, de la Chaux $R$ et al. Leitlinie: Therapie der obstruktiven Schlafapnoe des Erwachsenen. HNO 2009; 57: 1136-1156

30 Randerath WJ, Verbraecken J, Andreas $S$ et al. Non-CPAP-Therapies in obstructive sleep apnoea. Eur Respir J 2011; 37: 1000-1028

31 Peppard PE, Young T, Palta $M$ et al. Longitudinal study of moderate weight change and sleep-disordered breathing. JAMA 2000; 284: $3015-3021$

32 Newman $A B$, Foster G, Givelber $R$ et al. Progression and regression of sleep-disordered breathing with changes in weight: the Sleep Heart Health Study. Arch Intern Med 2005; 165: 2408-2413

33 Isono S. Obesity and obstructive sleep apnoea: mechanisms for increased collapsibility of the passive pharyngeal airway. Respirology 2012; 17: $32-42$

34 Morgenthaler TI, Kapen S, Lee-Chiong T et al. Practice parameters for the medical therapy of obstructive sleep apnea. Sleep 2006; 29: 1031 1035

35 Foster GD, Borradaile KE, Sanders $M H$ et al. A randomized study on the effect of weight loss on obstructive sleep apnea among obese patients with type 2 diabetes: the Sleep AHEAD study. Arch Intern Med 2009; 169: $1619-1626$

36 Tuomilehto HP, Seppa JM, Partinen MM et al. Lifestyle intervention with weight reduction: first-line treatment in mild obstructive sleep apnea. Am J Respir Crit Care Med 2009; 179: 320-327
37 Lam B, Sam K, Mok WY et al. Randomised study of three non-surgical treatments in mild to moderate obstructive sleep apnoea. Thorax 2007; 62: 354-359

38 Cartwright RD. Effect of sleep position on sleep apnea severity. Sleep 1984; 7: $110-114$

39 Oksenberg A, Silverberg DS, Arons $E$ et al. Positional vs nonpositional obstructive sleep apnea patients: anthropomorphic, nocturnal polysomnographic, and multiple sleep latency test data. Chest 1997; 112: 629-639

40 Oksenberg A, Silverberg $D$, Offenbach $D$ et al. Positional therapy for obstructive sleep apnea patients: A 6-month follow-up study. Laryngoscope 2006; 116: 1995-2000

41 Wenzel S, Smith E, Leiacker R et al. [Efficacy and longterm compliance of the vest preventing the supine position in patients with obstructive sleep apnea]. Laryngorhinootologie 2007; 86: 579-583

42 Maurer JT, Stuck BA, Hein G et al. [Treatment of obstructive sleep apnea with a new vest preventing the supine position]. Dtsch Med Wochenschr 2003; 128: $71-75$

43 Randerath WJ, Heise M, Hinz R et al. An individually adjustable oral appliance vs continuous positive airway pressure in mild-to-moderate obstructive sleep apnea syndrome. Chest 2002; 122: 569-575

44 Marklund M, Verbraecken J, Randerath W. Non-CPAP-Therapies in obstructive sleep apnoea mandibular advancement device therapy. Eur Respir J 2011; 37: 1000 - 1028

45 Chan AS, Sutherland K, Schwab RJ et al. The effect of mandibular advancement on upper airway structure in obstructive sleep apnoea. Thorax 2010; 65: 726 - 732

46 Kyung SH, Park YC, Pae EK. Obstructive sleep apnea patients with the oral appliance experience pharyngeal size and shape changes in three dimensions. Angle Orthod 2005; 75: 15-22

$47 \mathrm{Ng} \mathrm{AT}$, Gotsopoulos H, Qian J et al. Effect of oral appliance therapy on upper airway collapsibility in obstructive sleep apnea. Am J Respir Crit Care Med 2003; 168: $238-241$

48 Vanderveken OM, Devolder A, Marklund M et al. Comparison of a custom-made and a thermoplastic oral appliance for the treatment of mild sleep apnea. Am J Respir Crit Care Med 2008; 178: 197-202

49 Dort LC, Hadjuk E, Remmers JE. Mandibular advancement and obstructive sleep apnoea: a method for determining effective mandibular protrusion. Eur Respir J 2006; 27: 1003 - 1009

50 Kato J, Isono S, Tanaka A et al. Dose-dependent effects of mandibular advancement on pharyngeal mechanics and nocturnal oxygenation in patients with sleep-disordered breathing. Chest 2000; 117: 10651072

51 Ferguson KA, Cartwright $R$, Rogers $R$ et al. Oral appliances for snoring and obstructive sleep apnea: a review. Sleep 2006; 29: 244-262

52 Pitsis AJ, Darendeliler MA, Gotsopoulos $H$ et al. Effect of vertical dimension on efficacy of oral appliance therapy in obstructive sleep apnea. Am J Respir Crit Care Med 2002; 166: 860-864

53 Gotsopoulos H, Chen C, Qian J et al. Oral appliance therapy improves symptoms in obstructive sleep apnea: a randomized, controlled trial. Am J Respir Crit Care Med 2002; 166: 743-748

54 Marklund M, Stenlund H, Franklin KA. Mandibular advancement devices in 630 men and women with obstructive sleep apnea and snoring: tolerability and predictors of treatment success. Chest 2004; 125 : $1270-1278$

55 Hoekema A, Stegenga B, Wijkstra PJ et al. Obstructive sleep apnea therapy. J Dent Res 2008; 87: 882-887

56 Liu Y, Lowe AA, Fleetham JA et al. Cephalometric and physiologic predictors of the efficacy of an adjustable oral appliance for treating obstructive sleep apnea. Am J Orthod Dentofacial Orthop 2001; 120: 639-647

57 Ferguson KA, Ono T, Lowe AA et al. A short-term controlled trial of an adjustable oral appliance for the treatment of mild to moderate obstructive sleep apnoea. Thorax 1997; 52: 362 - 368

58 Ferguson $K A$, Ono T, Lowe AA et al. A randomized crossover study of an oral appliance vs nasal-continuous positive airway pressure in the treatment of mild-moderate obstructive sleep apnea. Chest 1996; 109: $1269-1275$

59 Engleman HM, McDonald JP, Graham D et al. Randomized crossover trial of two treatments for sleep apnea/hypopnea syndrome: continuous positive airway pressure and mandibular repositioning splint. Am J Respir Crit Care Med 2002; 166: 855-859

60 Barnes M, McEvoy RD, Banks $S$ et al. Efficacy of positive airway pressure and oral appliance in mild to moderate obstructive sleep apnea. Am J Respir Crit Care Med 2004; 170: 656-664 
61 Tan YK, L'Estrange PR, Luo YM et al. Mandibular advancement splints and continuous positive airway pressure in patients with obstructive sleep apnoea: a randomized cross-over trial. Eur J Orthod 2002; 24: $239-249$

62 Walker-Engstrom ML, Ringqvist I, Vestling 0 et al. A prospective randomized study comparing two different degrees of mandibular advancement with a dental appliance in treatment of severe obstructive sleep apnea. Sleep Breath 2003; 7: 119-130

63 Kushida CA, Morgenthaler TI, Littner MR et al. Practice parameters for the treatment of snoring and Obstructive Sleep Apnea with oral appliances: an update for 2005. Sleep 2006; 29: 240-243

64 Villa MP, Malagola C, Pagani J et al. Rapid maxillary expansion in children with obstructive sleep apnea syndrome: 12-month follow-up. Sleep Med 2007; 8: 128-134

65 Pirelli P, Saponara M, Guilleminault C. Rapid maxillary expansion in children with obstructive sleep apnea syndrome. Sleep 2004; 27: $761-766$

66 Villa MP, Rizzoli A, Miano $S$ et al. Efficacy of rapid maxillary expansion in children with obstructive sleep apnea syndrome: 36 months of follow-up. Sleep Breath 2011; 15: 179-184

67 Guilleminault C, Li KK. Maxillomandibular expansion for the treatment of sleep-disordered breathing: preliminary result. Laryngoscope 2004; 114: $893-896$

68 Guimaraes KC, Drager LF, Genta PR et al. Effects of oropharyngeal exercises on patients with moderate obstructive sleep apnea syndrome. Am J Respir Crit Care Med 2009; 179: 962 - 966

69 Puhan MA, Suarez A, Lo CC et al. Didgeridoo playing as alternative treatment for obstructive sleep apnoea syndrome: randomised controlled trial. BMJ 2006; 332: 266-270

70 Randerath WJ, Galetke W, Domanski $U$ et al. Tongue-muscle training by intraoral electrical neurostimulation in patients with obstructive sleep apnea. Sleep 2004; 27: 254-259

71 Schwartz AR, Eisele DW, Hari A et al. Electrical stimulation of the lingual musculature in obstructive sleep apnea. J Appl Physiol 1996; 81: $643-652$

72 Miki H, Hida W, Chonan T et al. Effects of submental electrical stimulation during sleep on upper airway patency in patients with obstructive sleep apnea. Am Rev Respir Dis 1989; 140: 1285-1289

73 Van de Heyning PH, Badr MS, Baskin JZ et al. Implanted upper airway stimulation device for obstructive sleep apnea. Laryngoscope 2012; 122: $1626-1633$

74 Friedman M, Tanyeri $H$, La RM et al. Clinical predictors of obstructive sleep apnea. Laryngoscope 1999; 109: 1901 -1907

75 Nakata S, Miyazaki S, Ohki $M$ et al. Reduced nasal resistance after simple tonsillectomy in patients with obstructive sleep apnea. Am J Rhinol 2007; 21: $192-195$

76 Nakata S, Noda A, Yanagi $E$ et al. Tonsil size and body mass index are important factors for efficacy of simple tonsillectomy in obstructive sleep apnoea syndrome. Clin Otolaryngol 2006; 31: 41 - 45

77 Won $\mathrm{CH}$, $\mathrm{Li}$ KK, Guilleminault C. Surgical treatment of obstructive sleep apnea: upper airway and maxillomandibular surgery. Proc Am Thorac Soc 2008; 5: $193-199$
78 Pirsig W, Schafer J, Yildiz F et al. [Uvulopalatopharyngoplasty without complications: a Fujita complication]. Laryngorhinootologie 1989; 68: $585-590$

79 Sher AE. Upper airway surgery for obstructive sleep apnea. Sleep Med Rev 2002; 6: 195-212

80 Elshaug AG, Moss JR, Southcott AM et al. Redefining success in airway surgery for obstructive sleep apnea: a meta analysis and synthesis of the evidence. Sleep 2007; 30: $461-467$

81 Walker-Engstrom ML, Tegelberg A, Wilhelmsson B et al. 4-year followup of treatment with dental appliance or uvulopalatopharyngoplasty in patients with obstructive sleep apnea: a randomized study. Chest 2002; 121: $739-746$

82 Kezirian EJ, Weaver EM, Yueh B et al. Incidence of serious complications after uvulopalatopharyngoplasty. Laryngoscope 2004; 114: 450-453

83 Friedman $M$, Schalch $P$, Lin HC et al. Palatal implants for the treatment of snoring and obstructive sleep apnea/hypopnea syndrome. Otolaryngol Head Neck Surg 2008; 138: 209-216

84 Woodson BT, Steward DL, Weaver EM et al. A randomized trial of temperature-controlled radiofrequency, continuous positive airway pressure, and placebo for obstructive sleep apnea syndrome. Otolaryngol Head Neck Surg 2003; 128: 848-861

85 Lin HC, Friedman M, Chang HW et al. The efficacy of multilevel surgery of the upper airway in adults with obstructive sleep apnea/hypopnea syndrome. Laryngoscope 2008; 118: 902 - 908

86 Hochban $W$, Brandenburg $U$, Peter $J H$. Surgical treatment of obstructive sleep apnea by maxillomandibular advancement. Sleep 1994; 17: 624-629

87 Prinsell JR. Maxillomandibular advancement surgery in a site-specific treatment approach for obstructive sleep apnea in 50 consecutive patients. Chest 1999; 116: 1519-1529

88 Conradt $R$, Hochban W, Heitmann J et al. Sleep fragmentation and daytime vigilance in patients with OSA treated by surgical maxillomandibular advancement compared to CPAP therapy. J Sleep Res 1998; 7: $217-223$

89 Li KK, Powell NB, Riley RW et al. Long-Term Results of Maxillomandibular Advancement Surgery. Sleep Breath 2000; 4: 137-140

90 Genecov DG, Barcelo CR, Steinberg D et al. Clinical experience with the application of distraction osteogenesis for airway obstruction. J Craniofac Surg 2009; 20: 1817 - 1821

91 Flores $R L$, Shetye PR, Zeitler D et al. Airway changes following Le Fort III distraction osteogenesis for syndromic craniosynostosis: a clinical and cephalometric study. Plast Reconstr Surg 2009; 124: 590-601

92 Wang X, Wang XX, Liang $C$ et al. Distraction osteogenesis in correction of micrognathia accompanying obstructive sleep apnea syndrome. Plast Reconstr Surg 2003; 112: $1549-1557$

$93 \mathrm{Li} K K$, Powell NB, Riley RW et al. Distraction osteogenesis in adult obstructive sleep apnea surgery: a preliminary report. J Oral Maxillofac Surg 2002; 60: 6-10

94 Fujita S. UPPP for sleep apnea and snoring. Ear Nose Throat J 1984; 63: $227-235$

95 Riley RW, Powell NB, Guilleminault C. Obstructive sleep apnea syndrome: a review of 306 consecutively treated surgical patients. Otolaryngol Head Neck Surg 1993; 108: 117-125 